\title{
Migración de la malla a la unión gastroesofágica: una complicación infrecuente de la reparación de una hernia hiatal gigante
}

\section{Mesh migration into the gastroesophageal junction: a rare complication of giant hiatal hernia repair}

\author{
Andrés Felipe Salazar, ${ }^{1}$ (1) Liliana Cuevas-López, ${ }^{2}$ (1) Ricardo Metke. ${ }^{3 *}$ (1)
}

\author{
Gacceso abierto \\ Citación: \\ Salazar AF, Cuevas-López L, Metke R. Migración \\ de la malla a la unión gastroesofágica: una \\ complicación infrecuente de la reparación \\ de una hernia hiatal gigante. Rev Colomb \\ Gastroenterol. 2021;36(3):399-403. https://doi. \\ org/10.22516/25007440.683 \\ Cirujano general, jefe del Departamento de \\ Cirugía General, Hospital Palermo. Bogotá, \\ Colombia. \\ 2 Cirujana general, Departamento de Cirugía \\ General, Hospital Universitario San Ignacio, \\ Facultad de Medicina, Pontificia Universidad \\ Javeriana. Bogotá, Colombia. \\ 3 Residente de Cirugía General, Departamento \\ de Cirugía General, Hospital Universitario \\ San Ignacio, Facultad de Medicina, Pontificia \\ Universidad Javeriana. Bogotá, Colombia. \\ *Correspondencia: Ricardo Metke \\ rmetke@javeriana.edu.co \\ Fecha recibido: $\quad 18 / 10 / 20$ \\ Fecha aceptado: $27 / 05 / 21$
}

\begin{abstract}
Resumen
El reparo de la hernia hiatal es un tema de debate debido a las posibles complicaciones asociadas que han cambiado a través de los años. En la literatura se reportan complicaciones asociadas al procedimiento hasta en un $30 \%$ de los casos. Las complicaciones diferentes a la recurrencia y a largo plazo son infrecuentes, reportadas en menos del $9 \%$ de los casos. La inclusión de la malla protésica en el esófago es una rara complicación y solo se han reportado pocos casos sobre esta. Entre los factores asociados a este desenlace se encuentran descritos: el material protésico, la técnica quirúrgica y la tensión de la malla sobre el tejido intervenido; sin embargo, es difícil establecer asociaciones directas de cada factor dado que la literatura actual solo cuenta con reportes de casos.

A continuación, se muestra el caso clínico de un paciente, quien, después de una reparación de hernia hiatal con malla, presenta la inclusión de material protésico en el esófago; se aborda el diagnóstico y el manejo de la misma.
\end{abstract}

\section{Palabras clave}

Hernia hiatal gigante, reparo de hernia hiatal, malla protésica, inclusión esofágica.

\section{Abstract}

Hiatal hernia repair has been a subject of debate due to the possible associated complications that have changed over the years. The literature reports up to $30 \%$ of cases with complications associated with the procedure. Complications other than recurrence and long-term complications are rare and reported in less than $9 \%$ of cases. The migration of the prosthetic mesh into the esophagus is a rare complication and only a few cases have been reported. The factors associated with this outcome include prosthetic material, surgical technique, and mesh tension on the intervened tissue. However, it is difficult to establish direct associations of each factor since the current literature has only case reports.

The following is a clinical case of a patient in whom the prosthetic material migrated into the esophagus after a hiatal hernia repair with mesh. The diagnosis and treatment offered are discussed.

\section{Keywords}

Giant hiatal hernia; Hiatal hernia repair; Prosthetic mesh; Esophageal migration.

\section{INTRODUCCIÓN}

La hernia hiatal es una patología frecuente en la población general; sin embargo, su incidencia reportada no excede el $5 \%$, dado que solo un bajo porcentaje de los pacientes presenta sintomatología asociada ${ }^{(1)}$. Esta sintomatología puede variar de acuerdo con el tamaño de la hernia y la severidad de la patología; las que llegan a presentar síntomas en su mayoría mejoran con tratamiento médico. 
De acuerdo con las guías de manejo de la Sociedad Americana de Cirujanos Gastrointestinales y de Endoscopia ${ }^{(2)}$, el manejo quirúrgico se realiza cuando no hay resolución de los síntomas con el manejo médico o por la presencia de una hernia hiatal gigante (definida como $>5$ $\mathrm{cm}$ ) sintomática, la cual representa aproximadamente un $5 \%$ de las hernias ${ }^{(3)}$. Otros autores sugieren tratar la hernia hiatal cuando se hace el diagnóstico, independiente de la presencia de síntomas, por el riesgo que existe de terminar presentándolos; esto se ha reportado hasta en un $30 \%$. Adicionalmente se menciona el riesgo de presentar complicaciones de la hernia y la morbilidad asociada a un procedimiento quirúrgico de urgencias (que puede aumentar hasta un $17 \%$ a $57 \%{ }^{(3-5)}$. A pesar de lo descrito, estas indicaciones todavía están en debate.

La corrección quirúrgica de esta patología puede variar entre una hiatoplastia con puntos simples de sutura monofilamento absorbible o la adición de una malla separadora de componentes, cuando el defecto es grande. El tipo de corrección quirúrgica es motivo de controversia en la literatura actual y algunos mencionan que depende del cirujano definir el uso o no de malla ${ }^{(6,7)}$; esto último basado en que la resolución de los síntomas reportada en la literatura es mayor al $50 \%$ con la colocación de la malla protésica separadora de componentes ${ }^{(8)}$, y en algunos estudios comparativos se menciona que la colocación de la misma puede disminuir el porcentaje de recurrencia de la hernia hiatal de un $42 \%$ a un $9 \%^{(1)}$. Sin embargo, el hecho de colocar una malla genera riesgo de presentar una inclusión del material protésico $^{(1,9)}$. Desafortunadamente, muchos de estos estudios tienen un seguimiento incompleto y los metaanálisis no son conclusivos respecto a la colocación de la malla de forma rutinaria ${ }^{(6,7)}$. Asimismo, lo reportado en relación con la diferencia en el uso de mallas biológicas o sintéticas para disminuir estas complicaciones no es claro al concluir algún beneficio de una sobre otra ${ }^{(10,11)}$.

Con respecto a las complicaciones asociadas al procedimiento, en la literatura se reporta principalmente la recurrencia hasta en un $30 \%$ de los $\operatorname{casos}^{(6,7)}$; las complicaciones diferentes a la recurrencia y a largo plazo son infrecuentes $y$ se reportan en menos del $9 \%$ de los $\operatorname{casos}^{(8)}$. Cuando se realiza hiatoplastia con malla separadora de componentes se presentan complicaciones en un bajo porcentaje, entre los cuales se pueden nombrar: neumotórax, lesión de estructuras adyacentes al hiato esofágico, sangrado, estenosis de la luz esofágica, reproducción, y, en menor proporción, migración de la malla protésica a la luz esofágica. Sobre la última complicación descrita solo hay series de caso en el mundo (en la literatura revisada solo se menciona una serie de casos) $)^{(12)}$.

A continuación, se presenta el caso de una migración de malla separadora de componentes a la luz esofágica; se mues- tra el abordaje inicial, diagnóstico y manejo definitivo con el que se obtuvo un desenlace clínico favorable, lo que tiene relevancia ante este tipo de complicación rara para la cual no hay algoritmos de manejo establecidos en la literatura.

\section{PRESENTACIÓN DEL CASO}

Un paciente masculino de 83 años con antecedente de hernia hiatal gigante sintomática, para lo cual se le practica una hiatoplastia con la colocación de una malla separadora de componentes más funduplicatura tipo Nissen. Su evolución clínica es adecuada.

El paciente reconsulta diez meses después por presentar un cuadro de evolución de disfagia progresiva para sólidos y líquidos, con un empeoramiento en los últimos cuatro meses anteriores a la consulta. Al ingreso se realiza una endoscopia de vías digestivas altas (EVDA), en la que se evidencia un cuerpo extraño altamente sugestivo de ser material protésico de malla no franqueable a $36 \mathrm{~cm}$ de la arcada dentaria. En la Figura 1 se muestra la imagen de la endoscopia inicial.

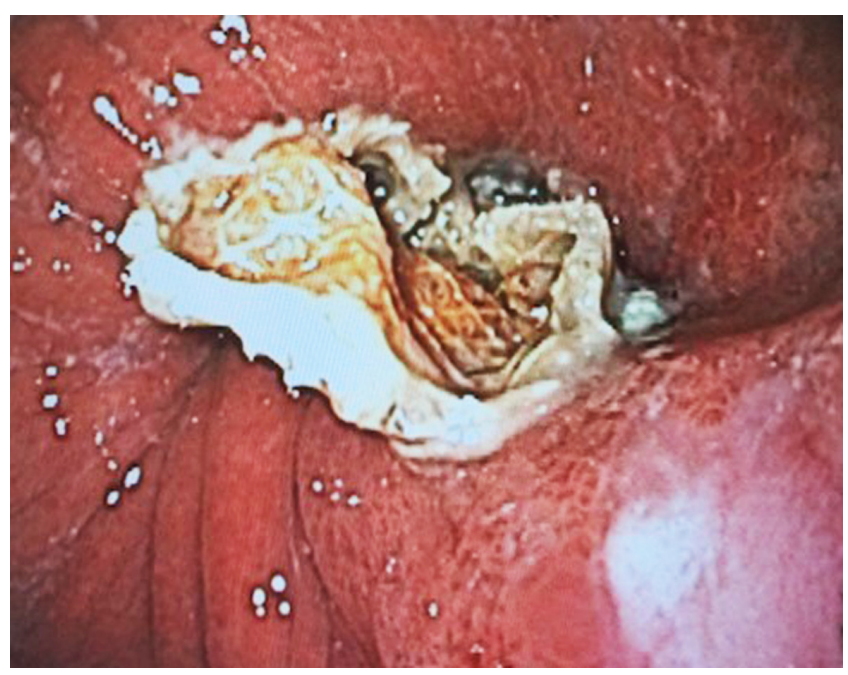

Figura 1. Imagen de la primera EVDA en la que se evidencia un cuerpo extraño altamente sugestivo de ser material protésico.

Ante la estabilidad del paciente, se decide hacer un estudio seriado de las vías digestivas altas, que evidencia el paso del medio de contraste sin reflujo, y una tomografía de tórax y de abdomen, que muestran cambios posquirúrgicos en la unión esofagogástrica con dilatación esofágica y retardo al paso de medio de contraste sin extravasación del mismo. En la Figura 2 se muestra el corte más representativo de la tomografía. Al no encontrar hallazgos que sugirieran perforación esofágica en los estudios practicados, se realizó una nueva endoscopia para intentar el corte y retiro de la malla. 


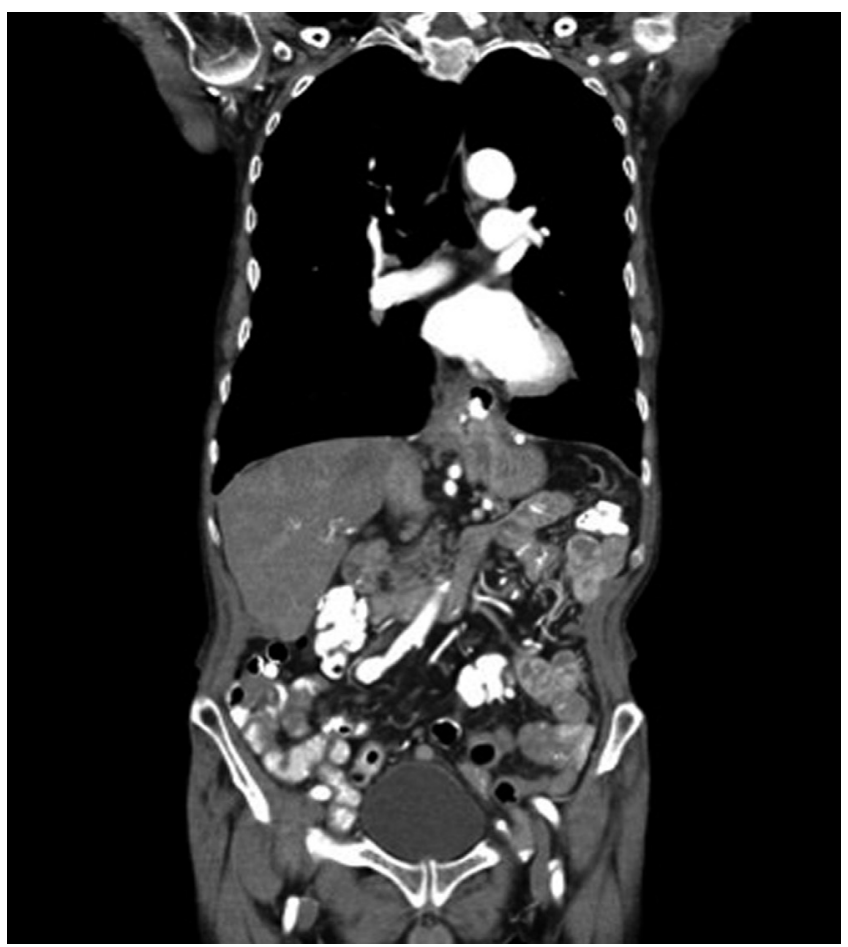

Figura 2. Imagen del corte coronal de la tomografía toracoabdominal, en la que se evidencian cambios posquirúrgicos en la unión esofagogástrica con dilatación esofágica sin extravasación del medio de contraste.

Se realizó una EVDA con un endoscopio de un solo canal. Se avanzó por el esófago hasta donde se evidenció cuerpo extraño sugestivo de malla, identificado previamente. Con una tijera endoscópica se realizó un corte del material en la luz esofágica con la posterior extracción de este. En total se requirió de dos sesiones endoscópicas; la primera sesión fue diagnóstica y la segunda terapéutica, logrando resecar y retirar la totalidad del segmento de la malla incluida. En la Figura 3 se muestra el segmento de la malla resecada.

Posterior a esto, el paciente presentó una evolución clínica y tolerancia a líquidos y sólidos adecuadas. En las consultas de seguimiento se evidenció una evolución clínica favorable durante los primeros meses. El paciente no asistió a controles posteriores. Durante la hospitalización inicial, el paciente autorizó y firmó el consentimiento informado para publicar el caso y las imágenes. Asimismo, la publicación del caso fue aprobada por el comité de investigación y de ética institucional.

\section{DISCUSIÓN}

La hernia hiatal es una patología frecuente muchas veces diagnosticada de forma incidental; en su mayoría, es

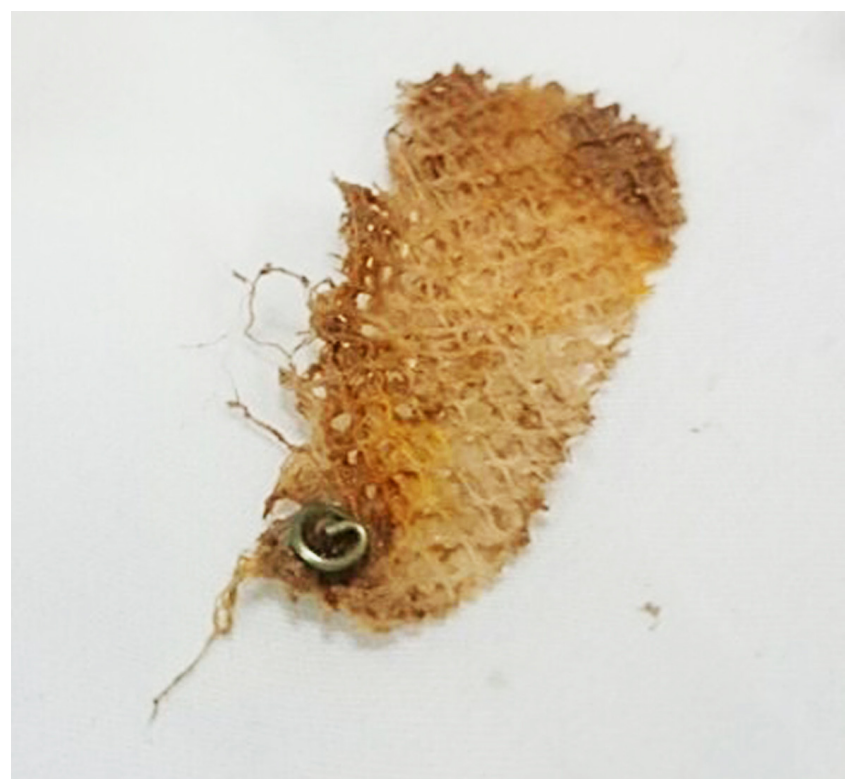

Figura 3. Segmento de la malla protésica resecado por endoscopia.

una hernia asintomática y cuando presenta síntomas usualmente mejora con el manejo médico. En el caso de la hernia hiatal gigante, por lo general es sintomática y tiene indicación de manejo quirúrgico; el tipo de procedimiento es debatido entre una hiatoplastia con o sin el uso de una malla debido a la alta tasa de recurrencia independiente del tratamiento quirúrgico y las complicaciones descritas ${ }^{(1)}$. Dentro de los resultados de los estudios y las recomendaciones de las guías de manejo de la Sociedad Americana de Cirujanos Gastrointestinales y de Endoscopia, la vía laparoscópica es de elección en la colocación de una malla protésica ${ }^{(2,9,13)}$. Respecto al tipo de malla, no hay una recomendación específica acerca del uso del tipo de material, como biológica sobre la no biológica. Algunos reportes sugieren un menor riesgo de inclusión con el uso de la primera; sin embargo, faltan estudios para generar una recomendación y por el momento se deja a criterio del cirujano ${ }^{(11)}$. En las guías de la Sociedad Americana de Cirujanos Gastrointestinales y de Endoscopia no se menciona el uso de la malla biológica ${ }^{(2)}$.

La inclusión esofágica de la malla es una complicación rara posterior a la corrección y la realización de la hiatoplastia con malla; la literatura la reporta en menos del $2 \%$ de los $\operatorname{casos}^{(11)}$. La principal manifestación clínica de este tipo de complicación es la disfagia, como en el caso de nuestro paciente. Una vez se realizan los estudios imagenológicos 
y endoscópicos que visualizan el componente de la malla intraluminal en el esófago distal, se realiza un intento de corrección de la complicación de manera no invasiva mediante endoscopia. Es importante mencionar en este aspecto la disponibilidad de estas medidas terapéuticas para poder hacer un manejo oportuno. Cuando el manejo endoscópico no es posible, se debe plantear un manejo quirúrgico para el retiro del material protésico con una esofagectomía distal con reconstrucción con anastomosis esofagogástrica ${ }^{(14)}$. En nuestro caso, el manejo endoscópico se pudo realizar de forma temprana, lo que favoreció esta opción terapéutica que finalmente condicionó un desenlace favorable. En caso de no poder realizar la extracción por endoscopia, se considera la posibilidad de realizar una corrección de manera quirúrgica; sin embargo, puede implicar un alto riesgo de morbilidad y mortalidad, por lo que no es la opción inicial.

\section{CONCLUSIÓN}

La inclusión esofágica de una malla es una complicación rara. El manejo inicial de esta complicación se puede hacer vía endoscopia con buenos desenlaces, como en este caso. En el seguimiento del paciente, si se encuentra que los síntomas persisten, se propone colocación de un stent esofágico y retirar el resto del material protésico en posteriores intentos endoscópicos. Se debe hacer un seguimiento estricto de estos pacientes, dado que una vez se presenta esta patología es posible que requiera manejos adicionales. En la actualidad no se ha instaurado un algoritmo que indique y estandarice los procedimientos para la corrección de esta complicación; sin embargo, bajo lo expuesto se considera que el manejo realizado puede representar una guía para el tratamiento adecuado en un paciente estable sin perforación esofágica, con buenos resultados.

\section{REFERENCIAS}

1. Mittal SK, Shah P. Current Readings: Failed Hiatal Hernia Repair. Semin Thorac Cardiovasc Surg. 2014;26(4):331-4. https://doi.org/10.1053/j.semtcvs.2015.01.002

2. Kohn GP, Price RR, DeMeester SR, Zehetner J, Muensterer OJ, Awad Z, Mittal SK, Richardson WS, Stefanidis D, Fanelli RD; SAGES Guidelines Committee. Guidelines for the management of hiatal hernia. Surg Endosc. 2013;27(12):4409-28. https://doi.org/10.1007/s00464-013-3173-3.

3. Chilintseva N, Brigand C, Meyer C, Rohr S. Chirurgie laparoscopique des hernies hiatales de grande taille avec renforcement prothétique de l'hiatus œsophagien. J Chir Viscerale. 2012;149(3):235-40. https://doi.org/10.1016/j.jchirv.2011.12.008

4. Braghetto I, Csendes A, Korn O, Musleh M, Lanzarini E, Saure A, Hananias B, Valladares H. Hernias hiatales: ¿cuándo y por qué deben ser operadas? [Hiatal hernias: why and how should they be surgically treated]. Cir Esp. 2013;91(7):438-43. https://doi.org/10.1016/j.cireng.2012.07.004

5. Prassas D, Rolfs TM, Schumacher FJ. Laparoscopic repair of giant hiatal hernia. A single center experience. Int J Surg. 2015;20:149-52. https://doi.org/10.1016/j.ijsu.2015.06.067

6. Sathasivam R, Bussa G, Viswanath Y, Obuobi RB, Gill T, Reddy A, Shanmugam V, Gilliam A, Thambi P. $<$ Mesh hiatal hernioplasty $>$ versus $<$ suture cruroplasty $>$ in laparoscopic para-oesophageal hernia surgery; a systematic review and meta-analysis. Asian J Surg. 2019;42(1):53-60. https://doi.org/10.1016/j.asjsur.2018.05.001
7. Tam V, Winger DG, Nason KS. A systematic review and meta-analysis of mesh vs suture cruroplasty in laparoscopic large hiatal hernia repair. Am J Surg. 2016;211(1):226-38. https://doi.org/10.1016/j.amjsurg.2015.07.007

8. Petersen LF, McChesney SL, Daly SC, Millikan KW, Myers JA, Luu MB. Permanent mesh results in long-term symptom improvement and patient satisfaction without increasing adverse outcomes in hiatal hernia repair. Am J Surg. 2014;207(3):445-8. https://doi.org/10.1016/j.amjsurg.2013.09.014

9. Oleynikov D, Jolley JM. Paraesophageal hernia. Surg Clin North Am. 2015;95(3):555-65. https://doi.org/10.1016/j.suc.2015.02.008

10. Rochefort M, Wee JO. Management of the Difficult Hiatal Hernia. Thorac Surg Clin. 2018;28(4):533-539. https://doi.org/10.1016/j.thorsurg.2018.07.001

11. Priego-Jiménez P, Salvador-Sanchís JL, Ángel V, Escrig-Sos J. Short-term results for laparoscopic repair of large paraesophageal hiatal hernias with Gore Bio $\mathrm{A}^{\circledR}$ mesh. Int J Surg. 2014;12(8):794-7. https://doi.org/10.1016/j.ijsu.2014.06.001

12. Jiménez AEP, Aguirre MEP, Campos AR, Eguizabal PT, Torres AJ, Serrano ID, Sánchez-Pernaute A. 658 - Management of Erosion of Hiatal Mesh into the Gastroesophageal Lumen. Gastroenterology. $2018 ; 154(6): S-1274$. https://doi.org/10.1016/S0016-5085(18)34186-6

13. Müller-Stich BP, Köninger J, Müller-Stich BH, Schäfer F, Warschkow R, Mehrabi A, Gutt CN. Laparoscopic meshaugmented hiatoplasty as a method to treat gastroesophageal reflux without fundoplication: single-center experience with 
306 consecutive patients. Am J Surg. 2009;198(1):17-24. https://doi.org/10.1016/j.amjsurg.2008.07.050

14. Coluccio G, Ponzio S, Ambu V, Tramontano R, Cuomo G. Dislocazione nel lume cardiale di protesi in PTFE utilizzata per il trattamento di voluminosa ernia jatale da scivola- mento. Descrizione di un caso clinico [Dislocation into the cardial lumen of a PTFE prosthesis used in the treatment of voluminous hiatal sliding hernia, A case report]. Minerva Chir. 2000;55(5):341-345. 